\title{
Optical path control of solitary waves in dye-doped nematic liquid crystals
}

\author{
Gaetano Assanto, ${ }^{1}$ Benjamin D. Skuse ${ }^{2}$ and Noel F. Smyth ${ }^{* 2}$ \\ ${ }^{I}$ NooEL - Nonlinear Optics and OptoElectronics Laboratory, Department of Electronic Engineering, University \\ "Roma Tre," Via della Vasca Navale 84, 00146 Rome, Italy \\ ${ }^{2}$ School of Mathematics and Maxwell Institute for Mathematical Sciences, The King's Buildings, University of \\ Edinburgh, Edinburgh, Scotland, U.K., EH9 3JZ
}

Received October 15, 2009; accepted December 22, 2009; published December 31, 2009

\begin{abstract}
The light-induced steering of spatial optical solitary waves in dye-doped nematic liquid crystals is analyzed. Shining a control beam through the cell results in a boundary mediated change in the orientation of the molecular director, thereby a perturbation in the refractive index of the medium. This re-orientational effect can optically steer spatial solitary waves propagating in an orthogonal plane within the liquid crystal layer. The equations governing this steering are solved using a Lagrangian formulation, resulting in modulation equations for the self-trapped beam path. Very good agreement is obtained between solutions of the modulation equations and numerical solutions of the full governing equations.
\end{abstract}

Nematicons- spatial optical solitary waves in nematic liquid crystals- result from a balance between linear diffraction and nonlinear self-focusing of an input beam in the presence of nonlocality, an effect brought about by the thermal or reorientational response of the material to light [1-2]. Once created, nematicons are stable and robust, with the ability to survive defects and perturbations [3-7]. An approach to introducing a defect into nematic liquid crystals (NLC) is to alter the response of the medium to light by introducing a small amount of dye molecules, a technique known as doping [8]. Dyedoping causes a change in the absorption of the NLC. In specific spectral intervals, an external illumination, i.e. a control beam, impinging onto the medium can perturb the molecular reorientation according to the control beam intensity and/or polarisation [9-11]. Whilst nematicons can survive the interaction with such defects, their evolution will be somehow affected, with a change in trajectory. Piccardi, Assanto, Lucchetti and Simoni [11] experimentally observed nematicons propagating in a dye-doped cell where control beams induced refractive defects; they demonstrated solitary wave steering dependent on power, shape and relative position of the external perturbation with respect to the nematicon. They also reported solitary wave refraction and total internal reflection [11]. In this Letter we investigate theoretically the steering of a nematicon by an optically driven refractive index change. Modulation equations describing

\footnotetext{
${ }^{*}$ E-mail: N.Smyth@ed.ac.uk
}

the beam path are derived and solutions of these equations are found to be in excellent agreement with numerical solutions of the governing equations.

Let us consider a linearly polarised, coherent light beam propagating through a cell filled with a dye-doped nematic liquid crystal (DD-NLC), as illustrated in Fig. 1. The input beam is launched so that it initially propagates at an arbitrary angle with respect to the direction $z$ down the cell. The coordinates $(x, y)$ are orthogonal to the $z$ direction. The input beam is polarised with its electric field along $x$, i.e. it is an extraordinary wave in the NLC equivalent uniaxial. In the bulk NLC the molecules are pre-tilted at an angle $\theta_{0}$ in the plane $(x, z)$ by an external electric field (voltage) across the thickness $x$. The beam injected at $z=0$ induces a further nonlinear rotation $\theta_{a}$ of the director [1]. In addition, a control beam between $z=z_{1}$ and $z=z_{2}$ is shone on the cell, as illustrated in Figure 1, with the resulting extraordinary refractive index $n_{e}$ shown in Fig. 2. As detailed in Ref. [11], this orthogonal beam

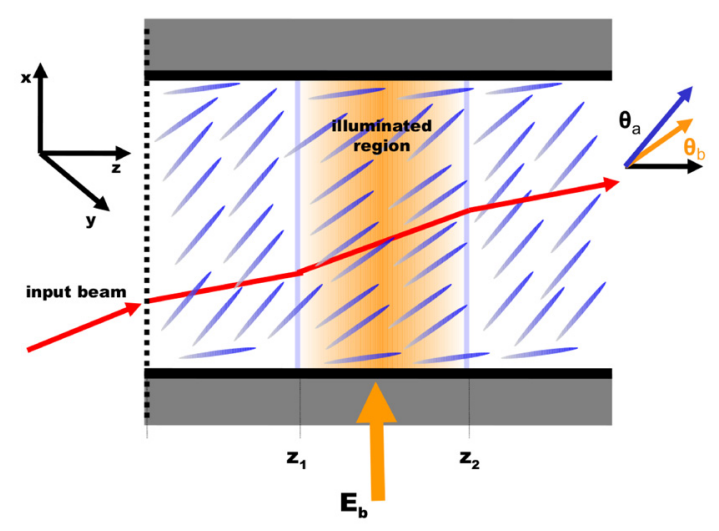

Fig. 1. Sketch of a dye-doped nematic liquid crystal cell with an externally illuminated region. The red line represents a nematicon with a curved trajectory due to the non-uniform index and optic axis distribution induced by the external beam. 


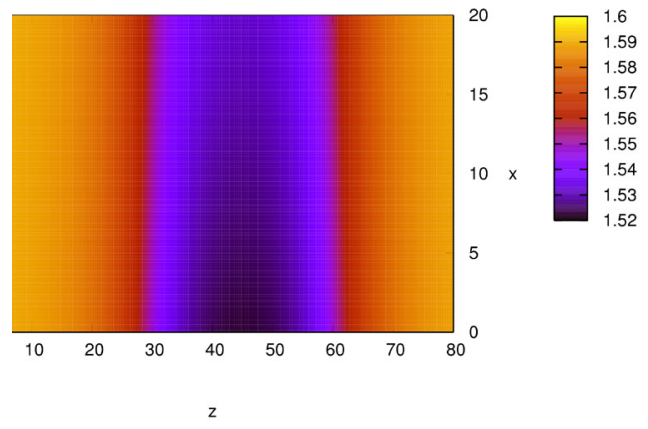

Fig. 2. Typical refractive index profile ne produced by a control beam.

can control the reorientation $\theta_{\mathrm{b}}$ of the director at the boundary by acting on the dye molecules there adsorbed, determining in turn the reorientation of the NLC volume under the illuminated region in the $x$ direction, with some decay and lateral spreading. Depending on its polarization, the control beam can cause a negative reorientation, with director rotation opposite to that induced by the input beam [11]; hence, the equation governing the propagation of the control beam through the NLC is [12]

$$
v \frac{\partial^{2} \theta_{b}}{\partial z^{2}}-2 \mathrm{q} \theta_{b}=2\left|E_{b}\right|^{2},
$$

where $E_{b}$ is the electric field envelope of the beam, $\mathrm{q}$ is related to the square of the static (or low frequency) field biasing and pre-tilting the NLC, $v$ measures the nonlocality of the medium, with a higher nonlocal response the larger $v$. Experiments are usually conducted in nonlocal NLC; hence, the large $v$ limit is the most appropriate regime to be considered here [13]. For a stripe shaped one-dimensional illumination $E_{b}$ is given by

$$
E_{b}=\left(\begin{array}{c}
E_{0,} z_{1}<z<z_{2} \\
0, \text { otherwise }
\end{array}\right) .
$$

The director distribution resulting from the external light control is then

$$
\theta_{b}=\left(\begin{array}{c}
A_{1} e^{\lambda z} e^{-\gamma x}, z<z_{1} \\
\left(A_{2} e^{-\lambda z}+A_{3} e^{\lambda z}-\frac{\left|E_{0}\right|^{2}}{q}\right) e^{-\gamma x}, z_{1}<z<z_{2} \\
A_{4} e^{-\lambda z} e^{-\gamma x}, z>z_{2}
\end{array}\right)
$$

where $\lambda=\sqrt{(2 \mathrm{q} / v)}$ and

$$
\begin{aligned}
& A_{1}=\frac{\left|E_{0}\right|^{2}}{2 \mathrm{q}}\left(e^{-\lambda z} 2-e^{-\lambda z} 1\right), A_{2}=\frac{\left|E_{0}\right|^{2}}{2 \mathrm{q}} e^{\lambda z} l \\
& A_{3}=\frac{\left|E_{0}\right|^{2}}{2 \mathrm{q}} e^{-\lambda z} 2, A_{4}=\frac{\left|E_{0}\right|^{2}}{2 \mathrm{q}}\left(e^{\lambda z} 1-e^{\lambda z} 2\right)
\end{aligned}
$$

As stated earlier, this externally induced all-optical reorientation is a boundary effect and the factor $\exp (-x)$ is introduced to account for its decay into the cell thickness [11]. The perturbation spreading in the plane $y z$ has been neglected to simplify the analysis.

The equations governing the propagation of the solitary beam are [12]

$$
\begin{gathered}
i \frac{\partial u}{\partial z}+\frac{1}{2} \nabla^{2} u+2 \theta_{a} u+2 \theta_{b} u=0, \\
v \nabla^{2} \theta_{a}-2 \mathrm{q} \theta_{a}=-2|u|^{2},
\end{gathered}
$$

where $u$ is the slowly varying electric field envelope of the launched beam and the Laplacians $\nabla^{2} u$ and $\nabla^{2} \theta_{a}$ are in the $(x, y)$ plane when the birefringent walk-off was ignored, taken out as a phase factor [14]. The director perturbation induced by the control beam acts as a refractive index distortion on the solitary wave path via the term $2 \theta_{b} u$. These equations have the corresponding Lagrangian formulation

$$
\begin{gathered}
L=i\left(\bar{u} u_{z}-u \bar{u}_{z}\right)-|\nabla u|^{2}+4\left(\theta_{a}+\theta_{b}\right)|u|^{2} \\
-v\left|\nabla \theta_{a}\right|^{2}-2 \mathrm{q} \theta_{a}^{2},
\end{gathered}
$$

where the over bar denotes the complex conjugate. To obtain approximate modulation equations describing the evolution of the nematicon we use the modulation theory approach of Minzoni, Smyth and Worthy [15]. The trial functions

$$
u=\operatorname{asech} \frac{\chi}{w} e^{i \psi}+i g e^{i \psi}, \theta_{a}=\alpha \operatorname{sech}^{2} \frac{\chi}{\beta},
$$

where

$$
\chi=\sqrt{(x-\xi)^{2}+y^{2}}, \psi=\sigma+V(x-\xi),
$$

are inserted into the averaged Lagrangian given by

$$
\bar{L}=\int_{-\infty}^{\infty} \int_{-\infty}^{\infty} L d x d y
$$

Here the electric field amplitude $a$, width $w$, director amplitude $\alpha$ and width $\beta$, nematicon position $\xi$ in $x$, transverse velocity $V$, phase $\sigma$ and height $g$ of the radiation shelf are functions of $z$. The modulation equations are obtained as variational equations from these parameters. Finally, diffractive radiative losses are calculated and included in the modulation equations in a similar manner to that of Kath and Smyth [16] and Minzoni, Smyth and Worthy [15].

A comparison between a full numerical solution and the solution of the modulation equations for the solitary wave amplitude and position is presented in Fig. 3. As expected on physical grounds, the nematicon propagates through the region with a non-uniform index distribution in $(x, z)$, i.e. with a non-straight trajectory. There is excellent agreement in the position of the beam peak and good agreement for the amplitude. 

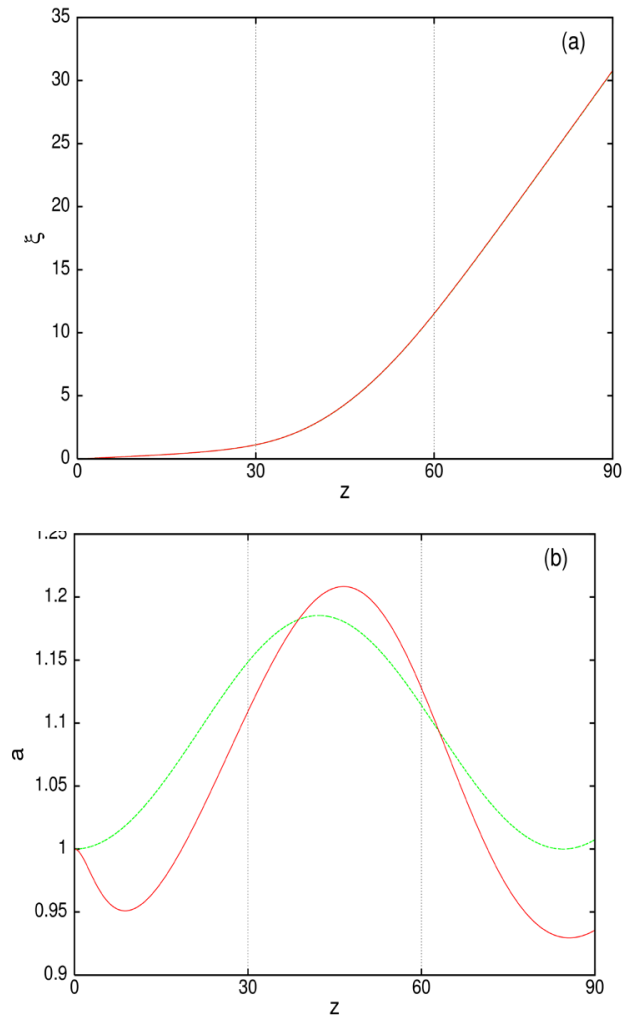

Fig. 3. Comparisons for the initial conditions. $a=1.0, w=3.5$, $V=0.0, \xi=0, E_{0}=1.5, \gamma=0.01, v=200$ and $q=2$ with $z_{l}=30$ and $z_{l}=60$. Full numerical solution (solid red); solution of modulation equations (green) (a) Positions (b) Amplitudes.

The self-trapped beam refracts even outside of the region where the control beam is shone, owing to the nonlocal response of the NLC, where reorientation is induced well outside the illuminated zone, as seen from the director solution (3) for $\theta_{b}$. It is remarkable that the approximate modulation equations model almost exactly the path deviation as given by the full governing equations. The accuracy of the modulation solution with respect to the angle of refraction for different initial $V$ values is shown in Fig. 4, using physical parameter values [17]. It is clear that the modulation solution is highly accurate for all values of $V_{0}$, particularly for values close to 0 . However, small differences begin to appear for large negative $V_{0}$ values. The most likely explanation for this small discrepancy is that the beam begins to experience large profile changes at higher $V_{0}$ values which cannot be modelled by a fixed ansatz beam profile. Nevertheless, the results presented reveal the utility of a variational approximation which reduces a system of partial differential equations with infinite degrees of freedom to a finite dimensional system of ordinary differential equations by which beam stability

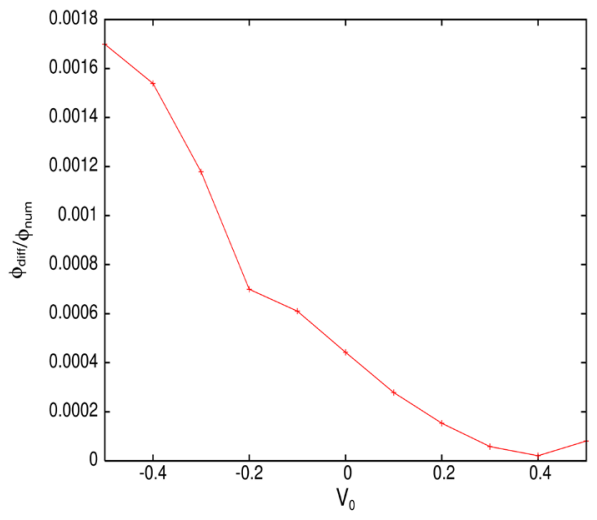

Fig. 4: Relative error in refraction angle, $\phi$, as a function of $V_{0}$ for input parameters $a=1.0, w=3.5, \xi=0.0, E_{0}=1.5, \gamma=0.01$, $v=200, q=2$, where $z_{1}=30$ and $z_{2}=60 . \phi_{\text {num }}$ is the numerically calculated refraction angle, $\phi_{\text {diff }}$ the difference between the modulation solution and $\phi_{\text {num }}$.

and dynamics can be investigated in more depth compared to a purely numerical approach. These methods are currently being employed in the study of other nematicon problems, including nematicon propagation in liquid crystal light valves.

This research was supported by the Royal Society of London under grant JP090179.

\section{References}

[1] M. Peccianti, G. Assanto, A. De Luca, C. Umeton and I. C. Khoo, Appl. Phys. Lett. 77, 7 (2000).

[2] G. Assanto, M. Peccianti, C. Conti, Opt. \& Photon. News 14, 45 (2003).

[3] M. Peccianti, C.Conti, G. Assanto, A. De Luca, C. Umeton, Nature 432, 733 (2004)

[4] M. Peccianti and G. Assanto, Phys. Rev. E 65, 035603R (2002)

[5] M. Peccianti, A. Dyadyusha, M. Kaczmarek and G. Assanto, Nat. Phys. 2, 737 (2006).

[6] S. V. Serak, N. V. Tabiryan, M. Peccianti and G. Assanto, IEEE Photon. Techn. Lett. 18, 1287 (2006).

[7] A. Pasquazi, A. Alberucci, M. Peccianti and G. Assanto, Appl. Phys. Lett. 87, 261104 (2005)

[8] I. C. Khoo, J. Park and J. Liou, Appl. Phys. Lett. 90, 151107 (2007).

[9] I. Janossy and A. D. Lloyd, Mol. Cryst. Liq. Cryst. 203, 77 (1991).

[10] A. Alberucci, G. Assanto, J. Nonl. Opt. Phys. Mat. 16, 295 (2007).

[11] A. Piccardi, G. Assanto, L. Lucchetti and F. Simoni, App. Phys. Lett. 93, 171104 (2008).

[12] C. Conti, M. Peccianti, G. Assanto, Phys. Rev. Lett. 91, 073901 (2003).

[13] C.Conti, M.Peccianti,G.Assanto, Phys. Rev. Lett. 92, 113902 (2004).

[14] B.D. Skuse and N.F. Smyth, Phys. Rev. A 79, 063806 (2009).

[15] A. A. Minzoni, N. F. Smyth, and A. L.Worthy, J. Opt. Soc. Am. B 24, 1549 (2007)

[16] W. L. Kath and N. F. Smyth, Phys. Rev. E 51, 1484 (1995).

[17] G. Assanto, A.A. Minzoni, M. Peccianti and N.F. Smyth, Phys. Rev. A 79, 033837 (2009). 